\title{
Enhancing the Multisensory Environment with Adaptive Game Audio Techniques
}

\author{
Ben Challis ${ }^{1}$, Angela Kang, Rachel Rimmer, and Mark Hildred ${ }^{2}$ \\ 1 Manchester Metropolitan University, Cheshire, UK \\ b.challis@mmu.ac.uk, \\ www.cheshire.mmu.ac.uk/dca/department-research \\ 2 Apollo Ensemble, York, UK \\ www. apolloensemble.co.uk
}

\begin{abstract}
Two workshop case studies are described that document the potential for applying novel approaches to the use of technology in multisensory environments. In contrast to current trends, the environments were regarded as a space within which to work rather than as a given set of technologies. Stimulating interactive story-worlds were enabled for groups of children with mixed Special Educational Needs where technology could empower the different groups to affect the environment as a whole. Arts-based leadership experience was regarded as key within the studies as were methods for moving beyond passive modes of interaction with sound and music. A novel approach to creating interactive 'soundtracks' is described that takes adaptive techniques from computer game audio and applies these within a physical space.
\end{abstract}

Key words: accessibility, music, dance, technology, multisensory environments, play, adaptive game audio

\section{Introduction}

It is widely accepted that there are observable benefits to be gained for individuals with significant learning difficulties who regularly interact with sensory stimuli. The suggestion that fundamental sensory stimulation could be a more direct way of reaching out to individuals with profound and multiple learning difficulties (PMLD) was originally proposed by Hulsegge and Verheul [1] in the late 1970s. In their book 'Snoezelen: Another World' they described specialist rooms within the De Hartenberg Centre in the Netherlands being equipped as controlled sensory environments where a care assistant could work with an individual with PMLD as she or he interacted with a range of sensory stimuli. There was great emphasis placed on reaction and play within these sessions and though, at some level, learning might be achieved, it was not a primary aim of the sensory activities.

The Snoezelen model of having dedicated sensory rooms (sometimes referred to as 'dark' and 'light' rooms) is still relatively commonplace within current Special Educational Needs (SEN) provision and there are now specialist suppliers that will equip such spaces. However, as Pagliano [2] observes, many of 
the technologies being used in the 1970s were becoming available as a result of the arrival of the discotheque where audio-visual equipment was emerging that would enhance the sensory environments being created for mainstream entertainment; mirror-balls, sound-to-light units and projector wheels were all commonly used against a backdrop of amplified and beat-based music. Alongside these audio-visual technologies, a variety of new plastic materials were also becoming available such that soft-play furnishings could be manufactured using wipe-clean PVC, velcro could be used for rapid but secure fastenings and vacuum forming techniques were enabling the production of lightweight playground equipment. There were also key sociological advances happening that would lead to a progressive movement away from the institutionalisation of individuals with physical and cognitive challenges and more towards mainstream integration. The Snoezelen concept emerged out of these landmark events, offering safe environments where individuals with PMLD could be immersed in stimulating yet playful activities and all within a therapeutic context.

The longterm benefits of working with sensory spaces are still to be fully assessed and where research has been carried out the results have tended to be inconclusive (e.g. [5, 3]) or perhaps not open to generalisation (e.g. [4]). There is still substantial value to be attached to personal observations and experiences though as the special needs educator is typically working at an individual needs level where the opportunity to generalise rarely arises. This is an aspect that Mount and Cavet [6] identify in their review of similar studies into the relative merits of multisensory environments (MSEs), ultimately arguing that there is likely to be as much significance to be placed on the quality and abilities of the individual member of staff as the equipment and spaces they are operating within. MSEs can now be regarded as widely available within SEN provision in the UK and have evolved from the Snoezelen model to exist in a number of contrasting forms including rooms, gardens, corridors, trolleys, pools and even corners. However, there is little literature available on what 'good' design practice might be or, indeed, the kinds of activities that might be carried out within any given environment. Recent research into the design and use of MSEs in England and Wales [7] has identified a number of areas that are worthy of further investigation.

Generic resources There is a noticeable trend for spaces to be equipped with a standardised set of resources (mirror-ball, bubble-tubes, infinity tunnel, audio playback etc.) yet with little evidence to suggest why this should be. In contrast, there is also evidence of SEN educators making creative use of repurposed technologies within ad hoc spaces with very positive results.

Passive use of sound and music Though there is evidence of individuals with PMLD responding positively to musical stimuli, much of the typical interaction with music in MSEs will tend to be passive; a backdrop against which other activities might be carried out.

Themed play and story-telling The technologies that typically populate MSEs are not always flexible in terms of enabling the creation of thematic environ- 
ments. Indeed such spaces can often be fixed environments with a standard set of resources.

Working with groups Although the Snoezelen model for the MSE was originally aimed at working exclusively with individuals with PMLD, there is now a wider recognition that exposure to sensory stimulation can offer potential benefits across a broader spectrum of people with learning difficulties and communication challenges; this can offer clearer opportunities for working with groups.

\section{Case studies}

Two case studies are described as initial attempts at addressing some of the issues that have just been outlined. They are also the start of a collaboration between the Department of Contemporary Arts (Manchester Metropolitan University) and Apollo Ensemble and it is anticipated that the project's findings will inform the design of new methods for interaction within specialist software environments. Both case studies have focused on the design of music workshops for children with cognitive and/or physical challenges attending SEN schools in the North of England and a number of common themes have been explored.

Environments Both sets of workshops were hosted in theatre 'black box' rehearsal spaces. This offered a relatively large space to work with such that a group of around fifteen individuals could work safely across the whole space if required. With black-out being available, any coloured lighting and projected images used could have greater impact, offering a more immersive sense of 'place' within the themed environments being suggested.

Technology A key aim was to create an immediately stimulating and almost 'magical' environment where simply being in the space would be fun or exciting. Added to this was the notion of being able to progress from one environment to another to create a journey or story to use as a backdrop for encouraging game play. To enable this, a core suite of wireless controllers was identified that could form the basis for rapidly establishing a themed environment or 'story world'. At the heart of this was an RFID card reading sensor that could be used to immediately switch from one environment to another with the participants being able to choose where to travel to next. The image printed on each card corresponded to a larger image being projected onto a backdrop screen along with an ambient soundscape that would complement that particular environment. In addition to this, coloured DMX lighting was being controlled to further enhance the immersive experience being created; blue for water, green for jungle, red for volcano etc. Colour changing LED spot lights were distributed on either side of the screen and partly around the workshop group in such a way that all lights could be switched to a specific colour simultaneously. Control over the colour of the lighting was achieved using a large but lightweight PVC dice housing an orientation sensor. Each side of the dice was a different colour and the lights 
were programmed to match the colour of that side which was face up; rotating the dice would rotate through the colours.

Game play As will already be apparent, great emphasis was placed on using game play throughout the workshops. Other than being a stimulating environment to experience in a passive sense it was important that the workshop should remain engaging throughout, offering opportunities for the group to make choices and to lead the way where possible. With this in mind, there was a careful balance to be maintained between prescribed and improvised activities such that there would always be a new activity to explore but wherever the opportunity might arise to react to an idea that emerged from the group it could be taken.

Feedback General observations were gathered that included reflections by workshop coordinators along with comments offered by educators and care-workers in attendance with each group. The input of these individuals was particularly valuable in terms of better understanding how stimulating and enjoyable the activities appeared to be for the groups with whom they were so familiar. They were also able to suggest how appropriate these same activities and environments might be for other groups that they were working with.

\subsection{Artscool 2015}

For this series of workshops, a story world was constructed around a tropical island adventure featuring ten locations including a beach, a jungle, rope bridges, paths, waterfalls, pools and a volcano. There were animal images that could be selected to appear on demand including an elephant, tiger, monkeys and parrots. Hand percussion was used to allow the group to create jungle rhythms throughout and carefully selected tuned percussion was used to allow pentatonic textures to be created at the beach and pool.

Simple rhythm-games were employed as ice-breaker activities at the opening of the workshop but the main focus from then on was the creation of a musical journey across the island moving between the different locations. There would be a starting location set by the workshop leaders but this choice could be passed to someone from the group by selecting a new card for the RFID reader; the new environment would appear automatically. Some time would be allowed to absorb the ambient soundscape and identify the sounds and images within it. Someone might use the dice sensor to pick a lighting colour to go with the scene and the activity could switch to playing musical games within the current location.

For the jungle images, the group might copy a simple rhythm to represent the sound of walking but then be ready to drop into a slower 'stomping' rhythm if the image of the elephant appeared on screen for example. The leaders would employ obvious physical gestures to suggest when to play loud or quiet, fast or slow, long or short and, finally, when to stop. At images of bridges, someone from the group might be asked to take a walk across the floor as if crossing a real bridge whilst the rest of the group would time their rhythm to match the footsteps. Walking steadily, pretending to teeter, becoming steady again and 
then a last dash to the end would all be supported by spontaneous but matching rhythms: steady, chaotic, steady, fast.

At the various waterside locations the musical activities would focus on creating serene textures with each member of the group having two or three selected chime bars to strike. The coordinator could conduct the group to gradually enter into the texture one-by-one, perhaps with a set rhythm or perhaps quite randomly. As with the rhythm games, the leaders would use physical gestures or body position to suggest whether the chimes should be struck loudly or quietly, quickly or slowly and shakers might be added to strengthen the water-like effect.

Reflections and observations Two groups of about ten children were involved with these workshops and, although the sessions were approximately one hour long, both groups remained completely engaged throughout and were clearly enthusiastic to take part. This was reinforced as an observation by both educators and care workers alike who made specific reference to these particular groups as being quite difficult to keep engaged; this level of sustained attention was far beyond their initial expectations. A key factor in this was probably the level of contrast being offered between the different locations where there was already much to absorb in terms of image, soundscape and lighting; simply changing locations would offer great variety and therefore new interest even in a quite passive sense. Participants were eager to contribute to the various musical games being led at each new location and opportunities to affect the environment directly were met with similar enthusiasm. Within the space, technology was being used to translate small movements into large gestures such that choices being made in a game-like way would dramatically affect the mood and feel of the immediate environment; rotating the dice would alter the colour of the whole environment and changing the image placed on the card-reader would transform the look and sound of landscape being visited. In this sense, the outcomes being offered were really quite empowering even though the interaction required for each was easy to achieve. Though the technology was enabling swift transitions between potentially exciting environments, the musical games and activities that were then explored were reliant on leadership from the workshop coordinators working with acoustic instruments. In this sense, the workshop design being explored here suggested a promising balance between technology enhanced environment and traditional music techniques but with considerable reliance on one or music specialists to lead and improvise around the activities.

\subsection{Cheshire Buddies 2015}

In contrast to Artscool, this workshop placed greater emphasis on the use of assistive technologies in triggering and controlling sound within the sensory environment. Again, efforts were made to create an exciting and magical atmosphere with images and soundscapes that could be used to create story-lines for game play. The design behind the workshop was tailored to allow for input from an experienced community dance practitioner. She would lead much of the gameplay within the environment, encouraging actions that would make use of body 
movement across the available space. The themed environments included outer space, walking on the moon, scuba diving, mountain climbing and skiing and as with the earlier workshops, each set of images would have unique soundscapes and controllable lighting to enhance the overall effect.

Additional technologies such as motion sensors and pressure sensitive floorpads were incorporated such that all the music supporting the dance could be shaped and controlled by the physical movement of the participants. To enable this, the music for the workshop was created using a compositional approach drawn from computer gaming that is generally described as adaptive audio. Such approaches can allow a constant soundtrack to adapt as the game-play follows a non-linear narrative. For example, a single parameter of 'intensity' might be used to match a game's levels of action, influencing the pace, texture and style of accompanying music in addition to any environmental ambiences, sound effects and musical stingers. Though there are several different approaches for creating adaptive audio environments (see [8]), one key approach is to create looped music as a series of layers that are always in play but where the overall audio-mix will be dependant on the changing value of just one or two parameters.

For the purposes of this workshop, a number of pieces of adaptive music were produced that could all be mapped and controlled using only a small number of parameters. To achieve this, each looped piece was based on six separate layers each of which had independent volume control. Two layers (typically bass and percussion) were controlled using a Leap Motion sensor (a non-contact desktop gaming device that monitors hand and finger gestures). This particular sensor was chosen knowing that there could be one or two individuals within the group with mobility challenges yet just simple left or right hand movement could enable these individuals to still take part and to contribute in a particularly effective way. The remaining four layers would be musically textural, made of harmonic patterns and incidental melodic phrases, all mapped along the single dimension offered by an ultrasound sensor with a range of about three metres. Though only offering a single dimension to work with, adaptive audio techniques mapped the same single value across four envelope parameters such that a variety of mixes could be moved through along the length of the beam. The aim was for one or two people to work with the beam, exploring the different layered mixes in an expressive way. Lastly, each of four floor-pad sensors could trigger a variety of 'stingers', again, a technique associated with game audio where a musical flourish can be triggered at any point in the game play whilst always appearing to 'fit' against the changing musical backdrop.

As with the earlier workshops, the structure behind the activities was partprescribed and part-improvised to once again enable the leader to respond and react to opportunities that might emerge from within the group. An initial icebreaker game involved the dice sensor being passed around the group in a circle. Each person created a dance gesture with the dice which would also effectively set the colour of the environment until the next person's turn. Though the same theatre black-box space was used for this workshop as with the previous ones, more lights were set up this time such that changing the colour offered 
even greater impact. Again, the card reader sensor was used to help choose the different locations and the leader would then look for body movement being suggested within the image to take the group on a journey across the floor space, imitating the movements of the leader. Once established as a game, the lead would be offered over to someone else in the group. Gradually, the three music controlling technologies were introduced to the group, once again playing and improvising through copying whilst creating the music which would support the activity for the rest of the group. Once all the different music making actions had been explored by the majority of the group a semi-improvised piece was devised that gradually involved more and more movement and music. Finally, some of the group members choreographed the actions of the others by indicating when and how to move.

Reflections and observations One group of approximately fourteen children took part in this particular workshop and similar levels of engagement and enthusiasm were observed throughout as with the earlier workshops. Again, this was noted by both the workshop coordinators and care workers alike. Though it is clearly positive that the environment was stimulating for the participants, the observations from the dance-coordinator leading the workshop are fundamental in terms of appreciating how the environment was suggesting and enabling particular modes of activity to take place. One of the most significant observations was that the environment and the technology within it were creating active opportunities for movement to occur. The environment provided an open structure to play within and because of this the role of the leader became one of facilitating participants towards being creative and playful on their terms, making choices about their engagement with the stimuli. Participants appeared to own their own movement and decisions with a sense of empowerment being created through the use of appropriate technologies; desires into active-opportunities into larger sonic and visual outcomes. The environment enabled play with no suggestion of 'right' or 'wrong'. Individuals could be encouraged to improvise and explore the technology which invited a range of responses and possibilities with every individual bringing something different into the space expressed though their own physicality.

\section{Conclusion}

Conceiving of the MSE as being a space within which to work as opposed to a discrete set of technologies can offer greater flexibility for creating stimulating interactive experiences. This additional flexibility can also enable group based activities to occur more readily and having specialist performance experience available enables improvisation to be used as a vehicle for creating and adapting activities to be both responsive and immediate. Using technology to enable actions and choices can clearly help make the environment accessible but the benefits of designing the environment to respond more coherently as a whole is perhaps less apparent; offering considerable empowerment by mapping small 
interactions into greater outcomes that transform the look, sound and 'feel' of the space. It is also apparent that devising an experience that is thematic can assist with the creation of a story-world to work within but that this can be further enhanced by conceiving of the story as being as a journey across a series of connected spaces. Lastly, applying adaptive techniques for controlling sound and music similar to those used in computer game design appears to offer intuitive interaction and exploration within a given sonic landscape. This allows artists to compose interactive soundtracks without knowing the exact gestures to be harnessed whilst also enabling workshop leaders who are perhaps musically inexperienced to lead group-based activities that offer expressive opportunities for interacting with music and sound. A simple model for employing adaptive audio techniques in MSEs has been demonstrated as a prototype and work is now under way to explore how best to enable this same concept within software tools such as Apollo Ensemble. Though the activities described here are regarded as novel, the participants for the case studies were all from SEN schools and in that sense can be regarded as relatively typical within a context of MSEs. However, there are likely to be other user groups who would benefit from engaging in sensory play where specific rehabilitative outcomes might be desired. With this in mind, the current project is being extended to working with stroke survivors where head-related trauma has led to often quite complex physical and cognitive challenges. The aim in this new context will be to consider how similar adaptive game-audio techniques might be mapped to quite specific individual movements and gestures that can complement a given programme of rehabilitation.

\section{References}

1. Hulsegge, J, Verheul, A.: Snoezelen: Another World, Rompa (1988)

2. Pagliano, P.: Using a multisensory environment: A practical guide for teachers, David Fulton Publishers (2001)

3. Stadele, N. D. and Malaney, L. A.: Effects of a multisensory environment on negative behavior and functional performance on individuals with autism. Journal of Undergraduate Research, IV (2001) pp. 211-218

4. Slevin, E. and McClelland, A.: Multisensory environments: are they therapeutic? A single-subject evaluation of the clinical effectiveness of a multisensory environment Journal of Clinical Nursing, 8 (1999) pp. 48-56

5. Vlaskamp, K., de Geeter, K. I., Huijsmans, L. M. and Smit, I. H.: Passive activities: The effectiveness of multisensory environments on the level of activity of individuals with profound multiple disabilities. Journal of Applied Research in Intellectual Disabilities, 16 (2003) pp. 135-143

6. Mount, H. and Cavet, J.: Multisensory environments: an exploration of their potential for young people with profound and multiple learning difficulties, British Journal of Special Education, 22(2) (1995) pp.52-55

7. Challis, B. P. Designing for Musical Play. In: Brooks, A. et al. (Eds.) Technologies of Inclusive Well-Being, Studies in Computational Intelligence 536, Springer-Verlag Berlin Heidelberg (2014) pp. 197-218

8. Van Geelen, T. Realizing Groundbreaking Adaptive Music. In: Collins, K. (Ed) From Pac Man to Pop Music. Ashgate. (2008) 